\title{
EL USO DE ENTONCES/ASÍ QUE EN CONTEXTO NARRATIVO SOBRE DATOS DEL PRESEEA-BUENOS AIRES
}

\author{
ClaUdia BORZI \\ Consejo Nacional de Investigaciones Científicas y Técnicas - Universidad de Buenos Aires \\ cborzi@filo.uba.ar
}

\begin{abstract}
Resumen
En este trabajo se estudian comparativamente los usos de entonces y de así que en el marco del Enfoque Cognitivo-Prototípico, que presupone que cada forma tiene un significado $\mathrm{y}$ una función propios, los que, a su vez, responden a distintas intenciones comunicativas del hablante. Siguiendo una metodología cualitativa y cuantitativa se analizan datos provenientes del nivel sociocultural alto del PRESEEA_BUENOS AIRES (Proyecto para el Estudio Sociolingüístico del Español de España y de América de la Ciudad de Buenos Aires) 2000-2010. Se identifica un contexto de uso particular para cada una de las formas: entonces en tematización de información y así que en abandono de turno. Se diferencian los contextos narrativos de los argumentativos. Se describe el uso más prototípico de cada una de las formas en el contexto narrativo. Se comparan los resultados con los obtenidos por Borzi (1997) sobre el cuerpo de datos oral de El Habla Culta de la Ciudad de Buenos Aires (PILEI) 1965-1969.
\end{abstract}

PALABRAS CLAVE: Enfoque cognitivo prototípico, PRESEEA_BAIRES, Marcadores, Conectores, entonces, así que.

\begin{abstract}
We compare the use of entonces and asi que in the framework of the Prototypical-cognitive view, which assumes that each form has its proper meaning and function. Meaning and function, which respond to the speaker's communicative intentions. Through a qualiquantitative methodology, we analyze data coming from a high socio-cultural level of the PRESEEA_BUENOS AIRES (Proyecto para el Estudio Sociolingüístico del Español de España $y$ de América de la Ciudad de Buenos Aires) 2000-2010. We identify a particular contextual use for each one of the forms: entonces with thematic information and asi que when abandoning a turn. We distinguish narrative and argumentative contexts. We describe the more prototypical use of each form in the narrative context. The results are compared with Borzi (1997), a former work performed on the oral data set El Habla Culta de la Ciudad de Buenos Aires (PILEI) 1965-1969.
\end{abstract}

KEY WORDS: Prototypical Cognitive Framework, PRESEEA_BAIRES, Markers, Connectors, entonces, así que.

\section{Introducción}

En el presente trabajo se estudian los usos de entonces y así que sobre parte del cuerpo de datos oral del Proyecto para el Estudio Sociolingüístico del Español de España y de América de la Ciudad de Buenos Aires 2000-2010 (PRESEEA-BUENOS AIRES). Se 
comparan los resultados con los obtenidos por Borzi (1997) sobre el cuerpo de datos de El Habla Culta de la Ciudad de Buenos Aires (HCBA) ${ }^{1}$ recolectado entre 1965 y 1969. Se reconsidera el estado de la cuestión respecto de estas formas en las gramáticas del español y en artículos especializados. El trabajo se desarrolla en el marco del Enfoque Cognitivo-Prototípico, en la línea de autores como Langacker (1987 y 1991) y Lakoff (1987), quienes, entre otros presupuestos teóricos, sostienen que todo uso es significativo y merece ser descrito, por lo que se espera que las formas bajo estudio tengan, cada una, significado particular y cumplan una función propia en cada contexto de uso, razón por la cual se estudian los contextos de cada forma de manera contrastiva tratando de justificar en dicha comparación la elección de una o de otra.

Se trabajan ejemplos como (1) y (2) que muestran contextos semejantes, en tanto existe una relación semántica de consecutividad en ambos casos, pero que a su vez abren la posibilidad de que elementos contextuales más específicos estén motivando uno u otro uso.

(1) y (2) Inf. -[...] cerraron todas las persianas, del departamento ENTONCES dice quédense quietos porque si alguien hace algo los mato a todos, nosotros vamos a bajar ahora, se ve que había una camioneta o algo esperándolos, y bueno y nos vamos ASÍ QUE vos sabés que se fueron y nos quedamos todos en la mesa sentados que nos podríamos haber asomado por el balcón y los veíamos, nada, no nos movíamos, viste quedamos un rato como en shock todos quietos hasta que bueno después llamamos a la policía. [PRESEEA_BAIRES SS 1/17]

En el trabajo no se tienen en cuenta casos como (3), donde la forma funciona como pronombre adverbial (García Fernández, 1999: \$48.1.3.3) que indica acontecimiento contemporáneo con otro o inmediatamente posterior (como en Ya no estaremos aquí para entonces - NGLE, 2009: $\$ 17.9 \mathrm{~h}-$ ).

(3) Enc. -iAlquilabas en el centro o alquilabas medio retirado?

Inf. -Siempre fuera, en... playas chiquititas, casi desconocidas, qué sé yo, Aguas Verdes que todavía es medio desconocida, en aquel ENTONCES era más desconocida. [PRESEEA_BAIRES JE 4]

\footnotetext{
${ }^{1}$ La transcripción de dicho cuerpo de datos es una selección de encuestas grabadas dentro del "Proyecto de estudio coordinado de la norma lingüística culta de las principales ciudades de Iberoamérica y de la Península Ibérica" patrocinado por el Programa Interamericano de Lingüística y Enseñanza de Idiomas (PILEI); materiales editados por la Dra. Ana María Barrenechea y publicados en 1987 por el Instituto de Filología y Literaturas Hispánicas "Dr. Amado Alonso" de la Facultad de Filosofía y Letras de la Universidad de Buenos Aires, en dos tomos, bajo el título El Habla Culta de la Ciudad de Buenos Aires. Materiales para su Estudio.
} 
Tampoco se analizan casos donde se mantiene el significado anafórico sin evocar un tiempo ni pretérito ni futuro en relación con la enunciación en sí, sino de futurohipotético, por lo que se puede postular (4) como un estadio intermedio entre el adverbio y el conector. Se descartan también casos en los que no se puede determinar la coherencia discursiva entre los conectados.

(4) Inf. - [...] yo creo lo primero que necesita es que haya conciencia urbanística en todo sentido, porque ENTONCES [EN ESE CASO] San Telmo también va a ser más limpio,... es bastante sucio. Esteh... en todo lo que hace a la urbanidad, eso es lo que más necesita San Telmo ahora. [PRESEEA_BAIRES JE10]

\section{El problema y el marco teórico}

Como adelantamos, los ejemplos (1) y (2) permiten preguntarnos si estos usos están en variación libre, es decir, si el hablante elige arbitrariamente una u otra forma sin tener la intención de manifestar un significado diferente con cada una de ellas y si en consecuencia estas son intercambiables en dichos contextos sin provocar cambio de significado o si no es así. Dado el presupuesto teórico enunciado en el apartado anterior, según el cual el hablante (consciente o inconscientemente) siempre dice lo que quiere decir, corresponde pensar que cada uso en cada mensaje persigue un objetivo comunicativo determinado, y que el hablante elige cada forma. La existencia de una elección plantea la pregunta: ¿Por qué usa a veces entonces y otras veces así que en contextos semántica y pragmáticamente semejantes?

Siendo que nos apoyamos en los principios de la Gramática Cognitiva, se mencionan a continuación algunos de los aspectos puntuales que se ponen a prueba con la presente investigación. En primer lugar, y coherentemente con lo previamente dicho, si sostenemos que cada forma en cada contexto tiene un significado y una función, desde un primer momento no coincidimos con aquellos autores que consideran que puede haber formas usadas como meras muletillas, es decir, sin significado (como por ejemplo sugieren Fuentes Rodríguez (1987: 144) y Domínguez (2010: 372) en relación con entonces).

En segundo lugar, como se sabe, el enfoque considera que las categorías tienen en general límites difusos y que los atributos de cada miembro de una categoría no están necesariamente todos presentes en todos los miembros de cada categoría ni lo están en la misma medida. Por el tema que se desarrolla en el presente trabajo se evalúa este presupuesto, porque, como veremos, ciertos atributos, a saber, la capacidad deíctica y anafórica y la temporalidad que presenta entonces, no están vigentes en la misma medi- 
da en todos los contextos, como ocurre por ejemplo en los entornos con tematización en comparación con los entornos continuativos y argumentativos.

En tercer lugar, se busca también aportar evidencia a favor de la propuesta de que las formas con significados o funciones semejantes sí se agrupan entre sí. Concepto que muchas teorías manejan de manera exactamente inversa, presuponiendo que dada una construcción, por economía, el hablante prefiere tener una sola forma para marcar determinado significado y no más de una (Cf. Pereira Scherre y Naro, 1991). Sin embargo, como se mostrará, entonces se agrupa con $y$ cuando marca, al igual que la conjunción copulativa, continuidad dentro de la narración.

Recordemos finalmente que los planteos teóricos de la Gramática Cognitiva, entre los que se encuentran los particularmente enunciados, imponen una metodología específica a las investigaciones llevadas a cabo dentro de dicho marco. En principio, se espera que se opere sobre usos auténticos contextualizados, porque el significado de una forma solamente se concreta dentro del mensaje en una situación particular de uso. Como se dijo, los ejemplos provienen de un cuerpo de datos auténtico, recolectado según estrictas pautas sociolingüísticas y los resultados se comparan con otro estudio también desarrollado sobre otro corpus de características semejantes, pero temporalmente anterior. Según el enfoque, también se espera se lleve a cabo un estudio no solamente cualitativo sino también cuantitativo, para lograr así una validación social. Digamos por último que de los análisis esperados para completar la descripción de una forma (comparación con la(s) otra(s) forma(s) con la(s) que varía en un contexto dado y consigo misma) en este caso se lleva a cabo la primera comparación y no en todos los contextos, dado que el contexto argumentativo, por su complejidad se deja pendiente para un futuro trabajo.

\section{Revisión del estado de la cuestión}

Para esta apretada reseña se toman como ejes dos problemas: qué significado atribuyen los autores a entonces y a así que y qué función les atribuyen, ya sea en el marco de la oración o del discurso, según qué autor se esté revisando.

Respecto de entonces todos los gramáticos (así por ejemplo Bello, 1980 [1847]); la Gramática de la Real Academia de 1931; Roca Pons (1960), R. Seco (1978); Alcina Franch y Blecua (1975), y más cerca en el tiempo la NGLE describen el significado temporal deíctico y anafórico manifestado en casos como entonces yo no estaba aquí o ya no estaremos aquí para entonces (NGLE, 2009: \$17.9g y h). Categorialmente lo clasifican como adverbio. Describen su contenido diciendo que la forma alude a un significado básico: temporal anafórico de tiempo pasado que puede llegar a mostrar posterioridad. 
Algunos autores lo caracterizan en oposición a ahora (como un no-ahora; así por ejemplo Roca Pons y García Fernández (1999)).

Los autores que siguen asignan a entonces no solamente significado temporal sino también lógico o consecutivo, así lo dicen tanto los diccionarios de la Real Academia de 1984, de Casares 1979, y de Moliner (1981), como Butt y Benjamin (1988), Matte Bon (1992), Veciana (1984), Cortés Rodríguez (1991), Fuentes Rodríguez (1987), Vigara Tauste, quien habla de un significado "aproximadamente" consecutivo (1992: 408) y Álvarez (1991, 1995 y 1999).

A pesar de que el Diccionario de la Academia de 1984 y Casares categorizan la forma en ambos usos (temporal y consecutivo) como adverbio, otros autores acompañando el significado consecutivo de entonces lo analizan como conjunción Moliner (1981), Butt y Benjamin (1988), Veciana (1984), Cortés Rodríguez (1991), Fuentes Rodríguez (1987), y más recientemente Zorraquino y Portolés (1999: \$63.3.3.11) y la NGLE (2009: $\$ 30.13 \tilde{n})$. Este significado consecutivo queda asociado en algunos casos a la proposición condicional, lo que deja en claro que están operando sobre el carácter argumentativo del contexto en el que tratan entonces y otras veces a la proposición causal. Fuentes Rodríguez, por ejemplo, nos dice que entonces sustituiría a toda la cláusula causal, por lo que a su entender esto es "un primer paso en el proceso de lo temporal a lo causativo" (1987: 142). La NGLE (2009: \$17.9.i) presenta entonces como conjunción, correlativa en la apódosis de una oración con prótasis condicional con si y se puede entender que la NGLE propone que entonces sustituye a la prótasis ${ }^{2}$. Otros autores proponen distinta clase de palabra para esta forma: para Roca Pons es un adverbio demostrativo que funciona como pronombre relativo con antecedente. Matte Bon lo llama "demostrativo" siguiendo la línea de Franch y Blecua.

Cortés Rodríguez y Fuentes Rodríguez asocian el valor consecutivo con la clase de palabra conector extra-oracional (lo hacen en la línea en que Koch y Oesterreicher (1990) se refieren a marcas de cesura superestructural —Gliederungssignale-). Cortés Rodríguez y Fuentes Rodríguez de la función conector extra-oracional llegan a la función expletiva. Fuentes Rodríguez propone una cadena de sucesivas derivaciones desde el uso adverbial temporal a la forma expletiva: adverbal > conector $>$ partícula continuativa > fático > muletilla. Este "valor fático" (o de continuación de turno) ocupa además a Gili Gaya (1961), Lope Blanch (1984), Koch y Oesterreicher (Turn-

\footnotetext{
${ }^{2}$ Nos dice "su valor ilativo proviene de su capacidad para hacer referencia a una prótasis condicional anterior y, en último extremo, de su condición de demostrativo: "Si Pedro tiene razón, entonces (= 'si Pedro tiene razón’) más vale que esperemos.” (2009: \$30.13ñ).
} 
Beibehaltung) y Vigara Tauste. Casado Velarde (1998: 65), presenta la forma entonces como marcador que permite mantener la atención "interlocutiva" caso que puede acercarse al fático de Fuentes y al de continuación de turno de Koch y Oesterreicher.

Butt y Benjamin y Koch y Oesterreicher mencionan casos de entonces en inicio de turno -Anfangs/Eroeffnungssignale -, relacionados con el cambio de hablante. En el presente trabajo se analizan usos semejantes, pero asociados con la tematización de información. En Borzi (1997) se tratan los casos con entonces en inicio de turno introduciendo conclusión interrogativa con intención confirmatoria.

Casado Velarde (1998: 65) presenta entonces como marcador textual consecutivo, entendiendo por "marcador" formas reguladas idiomáticamente en una lengua concreta para expresar una determinada función textual (1998: 61).

Álvarez no incluye entonces cuando trata los conectores de coordinación consecutiva (sí menciona así que), sino como adverbio en "función cohesiva", asociándolo con por lo tanto o en consecuencia. Los llama después "enlaces extra-oracionales" consecutivos (1999: 3793). Semánticamente, dice que tienen significados discursivos más amplios que las conjunciones coordinantes y significados periféricos, que no llega a desarrollar puntualmente. Ofrece una detallada descripción sintáctica y prosódica: libertad posicional, posible acumulación; carácter tónico y entorno entonacional marcado por pausas.

Zorraquino y Portolés ${ }^{3}$ incluyen entonces al tratar los marcadores discursivos en virtud de su posible autonomía ${ }^{4}$. Como conector consecutivo le reconocen un cierto sentido de consecuencia como hacía Fuentes Rodríguez. Mencionan las siguientes funciones a) relaciona un miembro del discurso anterior, aunque sin constituir su paráfrasis, b) aporta "nuevas informaciones sobre un tópico al que servirían de comentario" (1999: 4107). Consideramos digna de ser destacada la preocupación de Zorraquino y Portolés por la calidad de la información, porque, como se verá, el presente trabajo alude a la calidad de la información tanto en los contextos de tematización como en los narrativos. Por su parte, son muy interesantes las diferenciaciones de Vigara Tauste, quien opera sobre usos coloquiales; señala que "da nueva orientación o progresión de la in-

\footnotetext{
${ }^{3}$ Zorraquino y Portolés (1999: 4057) definen los marcadores del discurso como unidades lingüísticas invariables que no ejercen una función sintáctica en la predicación oracional cuya función en el discurso es guiar, de acuerdo con sus distintas propiedades morfosintácticas, semánticas y pragmáticas las inferencias que se realizan en la comunicación.

${ }^{4}$ Los autores mencionan un caso donde el interlocutor solicita al hablante la conclusión o explicación de lo que ha dicho: -Si dijéramos siempre la verdad no sería posible la convivencia//-¿Entonces...?// -Yo huyo de estos tipos que que te saludan diciendo: [...].
} 
formación" (1992: 409). Caracteriza entonces como ilativo y como marca de "vacilaciones" (1992: 251). La caracterización de un entonces ilativo, es compartida por Zorraquino y Portolés (1999: \$63.3.3.11) y por la NGLE (2009: \$30.13ñ). Vigara Tauste (1992: 126) también ejemplifica con casos cercanos a los que aquí se llama "tematizadores" aunque no los describe.

En interrogación (combinado o solo); acumulado con pero o con $y$, y tomando como antecedente las palabras del interlocutor entonces es descripto por Moliner (1981), Fernández Ramírez (1986: \$86) al tratar preguntas reflejas, por Zorraquino y Portolés (1999: \$63.3.3.11), y por Matte Bon, quien va más allá y se refiere al caso ¿Y entonces? ¿Qué pasó? (1992: 301) que incita a seguir hablando. No se trata el contexto interrogativo en el presente trabajo, aunque sí se estudió sobre el HCBA (Borzi, 1997: 134).

La apretada síntesis que antecede atestigua que distintos autores han detectado los usos que se tratan aquí en relación con entonces y, como se verá, el presente trabajo coincide con muchas de las apreciaciones previamente reseñadas. Quizás la caracterización de entonces en contexto de tematización esté aquí formulada con mayor claridad. Consideramos, sin embargo, que el presente trabajo se diferencia de los mencionados en que aquí se intenta, partiendo de una metodología no solo cualitativa sino también cuantitativa, dar una respuesta a cuál o cuáles de estas funciones son las más (y las menos) típicas en el uso de la forma y cuáles son los atributos de significado más salientes, para poder ir identificando, conforme la distribución cuantitativa, su significado y para qué la usa el hablante. Creemos que la descripción que se propone aquí ofrece la posibilidad de sistematizar los usos y organizar mejor la caracterización.

En cuanto a así que, los autores, atendiendo al contexto consecutivo en el que se usa, interpretan la relación de las cláusulas que conecta como una subordinación, probablemente por la presencia de la forma que. Así ocurre con Lenz (1935); Gili Gaya (1961), el Esbozo de la Real Academia, Butt y Benjamin (1988); M. Seco (1989) y Alcina Franch y Blecua (1975).

Hay sin embargo autores que consideran que entre las cláusulas conectadas por así que se establece una relación de coordinación. Así lo hacen Bello; la Gramática de la Academia de 1931 y Kovacci (1990), quien basa su decisión en la imposibilidad de acumular así que con $y$ o con $o$, condición que las gramáticas consideran definitoria para decidir si la forma es o no una conjunción coordinante.

\footnotetext{
${ }^{5}$ Los siguientes autores no tratan esta forma ni como pronombre, ni como adverbio ni como conector conjuntivo: Kany (1969); Pottier (1962); Beinhauer (1978).
} 
Los autores que atienden a los casos en que así que inicia oración, analizan este marcador como una unión extra-oracional (así lo hacen Gili Gaya (1961) y Vigara Tauste (1992) por ejemplo) o en ciertos contextos lo ven como una marca de expresividad, por ejemplo Vigara Tauste (1992: 120).

Zorraquino y Portolés no tratan la construcción así que sino la forma así cuando aparece sola como marcador conector consecutivo introduciendo la conclusión (1999: $\$ 63.3 .3 .10)$. Lo mismo hace la NGLE (2009: $\$ 30.13$ y $\$ 30.13 \mathrm{n}$ ) clasificando así, junto con entonces, entre los conectores discursivos adverbiales y mencionando su significado consecutivo. La NGLE señala finalmente que son también adverbios demostrativos ${ }^{6}$.

En resumen, los autores estudian entonces y así que por separado, sin contemplar que comparten contextos, hecho que ameritaría una comparación. En relación con así que, más allá de las diferencias en el tipo de relación sintáctica que asignan, no tienen en cuenta, por ejemplo, el contexto de abandono de turno. En relación con entonces y en cuanto al significado, priorizan el valor temporal que asocian al adverbio, aluden a un valor pronominal (anafórico) en segunda instancia y desde allí llegan al valor demostrativo (deíctico). El significado consecutivo lo asocian a otra clase de palabra, la conjunción. Admiten usos sin significado, planteo con el que no coincidimos y si bien ofrecen una gama amplísima de registros, no dan una descripción unificada de los contextos registrados. Cuando aluden a la calidad de la información de la cláusula que introduce se refieren a la presencia de información nueva, aunque no detectan los contextos de información tematizada. Por otro lado, la detallada descripción del comportamiento de la forma que ofrece Álvarez no está acompañada, lamentablemente, de una clara justificación.

\section{Hipótesis}

Frente a la riqueza de análisis que ofrece la bibliografía, la hipótesis particular que se defiende en el presente trabajo en relación con entonces es que, en la oralidad, activa no solamente su valor mostrativo temporal anafórico, valor semántico que fundamenta su uso en contexto de tematización de información, sino también activa la capacidad de mostración hacia adelante es decir que orienta la información de izquierda a derecha dentro de la Complicación en fragmentos narrativos. En cuanto a así que, se sostiene que, en la oralidad, constituye un límite, lo que justifica su uso como marca de abando-

${ }^{6}$ El Diccionario de Partículas del español (DPDE) de Briz, Pons Bordería y Portolés no trata las formas bajo estudio. 
no de turno y a su vez justifica su especialización en focalizar la Coda o Resolución en fragmentos narrativos, explotando de esta manera también su capacidad para marcar posterioridad (NGLE, 2009: \$23.16k) o catáfora (NGLE, 2009: \$17.9k).

\section{Metodología}

Se analizan ejemplos de 11 Entrevistas de Nivel Sociocultural Alto en 3 bandas etarias, que reúnen aproximadamente 6 horas 50 minutos de grabación, partiendo de la primera transcripción de las muestras del PRESEEA-BUENOS AIRES. Se tuvieron en cuenta, para el estudio de los contextos en los que varían entonces y así que, solamente aquellas entrevistas en las que el mismo hablante usa ambas formas bajo estudio, pensando que esos contextos presentan óptimas condiciones para estudiar la variación porque es el mismo hablante el que percibe las diferencias contextuales que favorecen el uso de una o de otra forma. Se lleva a cabo un análisis cualitativo y cuantitativo. En virtud del análisis del corpus se formulan variables dependientes e independientes, se las correlaciona en tablas de doble entrada y se consideran los valores absolutos y los porcentajes. Se aplican la prueba de significación estadística del chi cuadrado $\left(X^{2}\right)$ que permite comprobar si hay dependencia entre las variables y el valor de odds ratio (o.r.) que marca la importancia del factor independiente considerado. Se sigue así en lo fundamental a García (1985) y a Martínez (2009).

\section{Análisis}

Se identifican diferentes contextos de uso de entonces y de así que. Dos de ellos muestran una distribución contrastiva: el correspondiente a abandono de turno y el correspondiente a tematización de información. En el restante contexto estudiado, que atiende al género discursivo del fragmento con contexto consecutivo, las formas varían pudiéndose identificar características particulares que favorecen la elección de una o de la otra.

\subsection{Contexto conversacional de la entrevista: los turnos}

En el marco del intercambio conversacional de la entrevista semiestructurada que rodea a los usos bajo análisis, y de la negociación de los interlocutores (Briz, 1998), se encontraron casos en los que el hablante marca al interlocutor su intención de abandonar el turno, usando en particular una de las formas bajo análisis. El contexto de uso presentó en los ejemplos del corpus las siguientes características formales: a) Acumula- 
ción de partículas previas (véase, por ejemplo en (5), la presencia de $y$, claro, por supuesto previas a así que e incluso inmediatamente posteriores ${ }^{7}$; b) Presencia de pausas semilargas o largas en el entorno previo y siguiente inmediato ${ }^{8}$ incluso mediato (como se ve en (5)). En lo referente a las características de contenido, se evidencia un claro cambio o cierre del (sub)tópico de conversación que se ve en casi todos los casos, reforzado por la intervención inmediata del entrevistador, en general, con la formulación de una pregunta. En (5) se percibe el paso del tópico (el robo del celular que sufrió una jovencita) a un sub-tópico (la caracterización de la persona que le ofreció ayuda a la damnificada). Se agrega el ejemplo (6) con entonces, el único que se ajusta a las características de abandono de turno: la forma se da antecedida por pausa, en un entorno fonético semejante al de abandono y la entrevistadora interviene rápidamente formulando otra pregunta que propone un sub-tópico (las próximas vacaciones) aceptado por la encuestada'.

(5) -[el teléfono] funciona lo más bien, pero bueno no tiene ni camarita ni mp nada, ni nada

-Y está perfecto, funciona para qué...

-Sí las funciones para qué... y claro, por supuesto ASÍ QUE...

-El chico.... con el que estaba... el que la ayudó.

-Y ese pobre [...] [PRESEEA_BAIRES SS 1/10]

(6) - ¿Y por qué... ibas en micro, por qué no ibas en auto, por ejemplo?

-Bueno, en esa época porque no tenía auto, creo (risas), pero bueno, pero bueno, si no porque... igualmente aunque hubiese tenido un auto, no... porque como voy a la

\footnotetext{
${ }^{7}$ Resulta interesante ver en el siguiente caso cómo refuerza, el entrevistado, la intención de abandonar el turno, repitiendo así que y agregando una marca posterior, bueno:

-Por eso. Para mí que [los ladrones] tenían un dato porque justo no estaba.

-Viste que hacen...

-Ehhhh una inteligencia, claro, para mí sí, ASÍ QUE... ASÍ QUE bueno...

-¿Y en Mendoza cómo fue?

${ }^{8}$ El siguiente ejemplo presenta pausa media previa representada por coma: [...] y uno termina mimetizándose, también, termina sintiéndose tan incómodo como la persona que tiene al lado, ASÍ QUE ...

-Y más allá del Bicentenario, ¿solés festejar las fechas patrias? [PRESEEA-BAIRES S/S216]

${ }^{9}$ La oposición se plantea como [+abandono de turno] versus otros, porque se encontró solamente 1 caso en el que se puede pensar que entonces se está usando para mantener el turno:

-No, este tiene, este tiene pero, pero es viejo [el ascensor], sí, no sé de qué año será.

¿Y qué, y ENTONCES este... te gusta vivir ahí?

-Ehhhh me gusta pero me gustaría más vivir afuera. [PRESEEA_BAIRES SS 1/2]
} 
montaña a andar por la montaña al auto lo tendría que usar solo para llegar hasta el lugar y para volver nada más, ENTONCES...

-Y si pudieras elegir, ¿qué preferirías hacer las próximas vacaciones?

-Eh... Me gustaría hacer algún... algún viaje [...] [PRESEEA_BAIRES SS 1]

\begin{tabular}{|c|c|c|c|}
\hline & 1 Abandono de turno & 2 Otros & \\
\hline \multirow[t]{3}{*}{ A Asíque } & 20 & 80 & 100 \\
\hline & $20 \%$ & $80 \%$ & $100 \%$ \\
\hline & $95,24 \%$ & $33,90 \%$ & \\
\hline \multirow[t]{5}{*}{ B Entonces } & 1 & 156 & 157 \\
\hline & $0,64 \%$ & $99,36 \%$ & $100 \%$ \\
\hline & $4,76 \%$ & $66,10 \%$ & \\
\hline & 21 & 236 & 257 \\
\hline & $100 \%$ & $100 \%$ & \\
\hline
\end{tabular}

Odds ratio $=39$

Tabla 1. Contexto de abandono de turno

La lectura vertical de la columna 1 de la Tabla 1 muestra una clara tendencia de así que como marca de abandono de turno (95,24\%, 20/21 de así que versus solamente un $4,76 \%, 1 / 21$ de entonces). En consonancia con los resultados obtenidos sobre el PRESEEA_BAIRES, en el HCBA, de entre todos los casos que marcaban abandono de turno, un 94,74\%, 18/19 de casos tenía así que y solamente un 5,26\%, 1/19 entonces. Como se advierte, los porcentajes son muy similares. Por su parte, la lectura horizontal de la Tabla 1 muestra un porcentaje interesante (Celda A1 20\%, 20/100) de entre todos los casos de así que en esta función, que explota la consonancia de este conector en contextos que marcan límite ${ }^{10}$. En el HCBA, sobre el total de casos de así que se encontró un 14,88\%, 18/121 marcando abandono de turno, se dan porcentajes también cercanos.

${ }^{10}$ El Diccionario de la Real Academia de 1984; Casares y el Esbozo (1973: \$3.21.3.c) aluden a un significado temporal de así que (Así que escuchaba mentar la palabra tisis, desfallecía), que no se registró ni en el HCBA ni en PRESEEA, pero que resulta muy interesante porque indica un límite temporal entre dos hechos y es un significado que puede verse como fundamento de la tendencia a participar de los contextos de abandono de turno y de cesura superestructural, como se tratará más adelante. 


\subsection{Contexto conversacional: la información}

Se considera en este caso el tipo de información de la cláusula que introducen las formas bajo análisis, algo que tiende a repercutir en la posición de dicha cláusula en el contexto conversacional.

Siguiendo básicamente los principios de la Escuela de Praga (Firbas, 1992) se identifican distintos tipos de información conocida (o accesible): aquella que ha sido mencionada de manera literal en el discurso previo (como ocurre en (7)); aquella información no mencionada literalmente con anterioridad, pero que el hablante evalúa como que puede ser inferida partiendo del conocimiento enciclopédico compartido con el interlocutor $^{11}$; aquella información que el interlocutor puede inferir (deducir) del contexto lingüístico previo sin necesidad de apelar a información enciclopédica específica ${ }^{12}$, o aquella información evidente, de inmediata interpretación porque lo designado está presente en la situación de enunciación ${ }^{13}$.

Respecto de la información nueva (o inaccesible) reconocemos los siguientes subtipos: información nueva porque el hablante no identifica el objeto designado en el momento de la enunciación, aunque presupone su existencia (es decir información nueva de manera absoluta para hablante y oyente) ${ }^{14}$; o información nueva porque no ha sido mencionada de manera (relativamente) literal en el discurso previo; o porque (el hablante cree que) el interlocutor no conoce el objeto ${ }^{15}$, o nueva porque el interlocutor

\footnotetext{
${ }^{11}$ En el siguiente ejemplo, ambos hablantes trabajan en el ámbito científico y su conocimiento enciclopédico les permite deducir que publicaciones importantes alude a publicaciones en revistas internacionales y a revistas indexadas:

-Dos en el período, de esos tres años, estamos hablando de publicaciones importantes, quiere decir...

-Ah, en revistas internacionales.

-... entonces en revistas indexadas, ¿no? Revistas que estén en Scielo que estén en Latindex. [PRESEEA_BAIRES SS218]

${ }^{12}$ Como en el siguiente ejemplo, donde la información subrayada, que sigue a entonces, se puede inferir del contexto lingüístico previo:

- ¿En la casa anterior tenías buena relación con los vecinos o son medio anónimos?

-Eh... yo soy anónima para ellos porque no estoy en todo el día en mi casa ENTONCES ellos me ven entrar de vez en cuando... [PRESEEA_BAIRES JS6]

${ }^{13}$ Como ocurre en el siguiente caso donde tenemos $1^{\circ}$ persona del singular nominativa y presente actual en la cláusula que sigue al conector: [...] funciona como un pueblo chiquito, ENTONCES yo pienso que voy a extrañar por todo el aspecto cultural que tiene Buenos Aires. [PRESEEA_BAIRES JE8].

${ }^{14}$ Como ocurre en Se lo preguntaré al estudiante que sepa de informática.

${ }^{15}$ Como ocurre en Se lo preguntaré a un estudiante que sabe de informática.
} 
no puede deducir la información ni del conocimiento enciclopédico ni de lo previamente dicho, como se da en (7).

En este caso se presentó pertinente la presencia/ausencia de información conocida por mencionada en el discurso previo que era introducida por el conector, la que al ser retomada casi literalmente permite decir que la forma entonces participa en la tematización de información a partir de la cual se continúa luego con una predicación. En (7), después de una digresión de la encuestada y de una intervención de la encuestadora, la encuestada retoma el turno y reinicia el relato en donde lo había abandonado. Entonces recupera información conocida, la tematiza (alude a la ausencia de respuesta por parte de quien entraba) que sirve de punto de partida para la introducción de otra información nueva para el interlocutor (abrió la puerta - entró con cuatro [personas] más). Se destaca en el ejemplo el texto en cuestión. Se registraron 17 casos en este contexto de tematización, un $10,83 \%, 17 / 157$, un porcentaje un tanto menor que el registrado en el HCBA, aunque cercano, que era de un 16,66\%, 37/222.

(7) -¿Cómo fue eso que te robaron?

- Y Jerónimo llegaba de la facultad a la noche y [...] llegaba de la facultad, y entonces oigo la llave y le digo Jerónimo ¿sos vos?, ¿Jerónimo? y no me contestaba, por eso ahora, te digo que cada vez que abren la puerta tengo que preguntar inmediatamente quién es y que alguien me responda rápido porque te juro que es lo único que me quedó esa impresión.

-Te quedó un trauma...

-ENTONCES Jerónimo no contestaba, cuando abrió la puerta en un momento entró con cuatro más y por un momento yo pensé que eran compañeros de la facultad. [PRESEEA_BAIRES SS 1/6]

Vigara Tauste ejemplifica casos semejantes sin describirlos en el sentido en que se hace aquí (1992: 126). Koch y Oesterreicher (1990) tratan casos semejantes como Anfangs-/Eröffnungssignale en cambio de turno. Aquí se corrobora que, aunque en ciertos ejemplos se da dicho cambio de turno, como en (7), no es necesario que haya cambio de turno para que haya tematización, lo que se advierte en (8):

(8) $-i Y$ en Egipto viste las Pirámides?

-Y en Egipto fuimos a las Pirámides, anduvimos en camello, pero ahí ya fue como un shock cultural, te digo que es todo tan diferente que... Fue el lugar que tuvimos como un cimbronazo porque la comida, la escri..., todo, todo es distinto...

-¿Cómo es?

-Diferente. ENTONCES ahí por ejemplo no pudimos quedarnos en un albergue. [PRESEEA SS 1/1] 
Así que no se da en contextos de este tipo. Seguidamente se presenta un ejemplo en el que, aunque la información que sigue al conector es información previamente mencionada (si bien no totalmente literal), no podemos pensar que se trate de una tematización sino que la analizamos como una información ecoica que cierra lo previamente dicho. En (9) se ve claramente que la encuestadora inicia otro par con una pregunta que se vuelve retórica en el contexto, de orientación negativa: ¿espectáculos al aire libre? $\mathrm{No}^{16}$. Se registraron 6 casos de así que $(6 \%, 6 / 100)$ en este contexto eco de cierre de un tópico o sub-tópico; un porcentaje mayor que el registrado en el HCBA, donde había solo un 2,64\%,1/38 de casos de así que en un contexto semejante.

(9) -Porque bueno es, justamente es un tipo, es un espectáculo visual, por lo tanto verlo en cine, digo, es una experiencia absolutamente distinta que bajarse el video...

[...]

-Y bueno, la verdad es que es espectacular lo que han logrado.

-Mjm, qué cosa. ASÍ QUE preferís ir al cine, antes que... ¿espectáculos al aire libre? No.

-No, hace muchísimos años que no voy... [PRESEEA_BAIRES SS3]

\subsection{Género del fragmento en el contexto conversacional de la entrevista}

En el transcurso de una entrevista se dan momentos donde en un mismo turno o en la cadena cooperativa de turnos tienen lugar fragmentos de intención más narrativa y otros de intención más argumentativa ${ }^{17}$. Este criterio contextual no ha sido tenido en cuenta por los gramáticos como definitorio cuando describieron el uso de estos dos conectores, aunque sí se han ocupado de entonces autores que estudian marcadores del discurso en particular. Así Domínguez (2010: 370-371) dice que entonces "organiza la narración" y en nota 13 , frente a un caso particular ${ }^{18}$ agrega que aporta en ese caso al

\footnotetext{
${ }^{16}$ La NGLE contempla usos semejantes a este que clasifica como conjunción ilativa que inicia período e incluso turno discursivo. Su ejemplo: ¿Así que encontraste a Neno acostado con tu mujer? (2009: 46.12.1)

${ }^{17}$ Asumimos, como recientemente retoma Domínguez (2010: 365), que los textos no son homogéneos en lo referente al tipo textual y que en un discurso conversacional se espera que se sucedan fragmentos narrativos, argumentativos y descriptivos sin que haya una explícita señal.

${ }^{18}$ El ejemplo (3) de la autora dice "Quedaron en escaparse en un tren de las seis de la tarde/creo que era/ y no apareció// Entonces él se fue a Casablanca/ empezó a vivir su vida" En el presente trabajo y dada la intención claramente narrativa del fragmento donde se encuentra el ejemplo citado se analizan
} 
enunciado una doble instrucción, ordenadora y argumentativa. Más adelante, a propósito del mismo ejemplo, agrega que es "marcador de la progresión temporal de la narración" (2010: 373). La autora no menciona ni así que ni entonces como marcadores propios de argumentaciones (2010: \$3.4), aunque sí da un ejemplo de entonces en un fragmento argumentativo (2010: 373) en el marco de textos narrativos. Como la autora no cuantifica ni compara de manera sistemática los tipos textuales entre sí ni las dos formas dentro de un mismo tipo textual no resulta del todo claro el criterio que permite decidir cuáles son conectores que pueden ser considerados propios de uno o de otro tipo textual.

Como se aprecia en la Tabla 2 que sigue, el género del fragmento se presenta como un atributo contextual de influencia en la elección de una u otra forma dentro de una conexión consecutiva entre las cláusulas. Siguen ejemplos donde prevalece la intención narrativa, casos (10) y (11) que ejemplifican las celdas A1 y B1 de la Tabla 2 respectivamente y otros donde prevalece la intención argumentativa, casos (12) y (13) que ejemplifican respectivamente las celdas A2 y B2 de la misma tabla.

(10) -[...] Poco tiempo, lo que pasa que como allá quedaron mis abuelos yo iba, iba todos los veranos, ya de chico iba allá, ENTONCES me encontraba con el carnaval y los corsos que se hacian en la calle San Martín y... y bueno y yo me disfrazaba también. [PRESEEA_BAIRES JM5]

(11) - ¿Qué hicieron?

-Eh nos fuimos a un hotel, y nos quedamos unos días, después fuimos a la embajada, nos conectamos con una gente de la embajada ASÍ QUE salíamos con unas chicas de la embajada, que nos llevaron a un club ahí en El Cairo, [...] [PRESEEA-BAIRES SS13]

(12) -[...] la verdad que no hizo nada al respecto; y la policía metropolitana no está cuando se la necesita, y están ganando sueldos por arriba de los cinco mil pesos, cuando los docentes no llegan ni a los dos mil doscientos; ENTONCES me parece que tenía que haber mejorado más la educación pública y además el la... [PRESEEA_BAIRES SS48]

(13) -[... ¿ ¿cómo te imaginás que hubiese sido tu vida, sin [la carrera en] sistemas?

-Eh... Creo que hubiese... me las... me hubiese... eh adaptado, a la... creo que a sistemas me adapté, no es que lo siento mi vocación ASİ QUE creo que me hubiese adaptado de la misma manera. [PRESEEA_BAIRES JD1]

estos contextos como continuativos por presentar causa/resultado. Los casos que quedan clasificados como argumentativos presentan un entorno de justificación/consecuencia. 


\begin{tabular}{|c|c|c|c|}
\hline & 1 Narrativo & 2 Argumentativo & \\
\hline \multirow[t]{3}{*}{ A Entonces } & 87 & 52 & 139 \\
\hline & $67,59 \%$ & $37,41 \%$ & $100 \%$ \\
\hline & $76,99 \%$ & $52 \%$ & \\
\hline \multirow[t]{5}{*}{ B Asíque } & 26 & 48 & 74 \\
\hline & $35,14 \%$ & $64,86 \%$ & $100 \%$ \\
\hline & $23,01 \%$ & $48 \%$ & \\
\hline & 113 & 100 & 213 \\
\hline & $100 \%$ & $100 \%$ & \\
\hline
\end{tabular}

Odds ratio: $3 X^{2}: 4,41(p>0,05)$

Tabla 2. Contexto de fragmento narrativo versus contexto de fragmento argumentativo

De la lectura vertical de la columna 1 de la Tabla 2 se puede concluir que el contexto narrativo favorece claramente la elección de entonces en lugar de así que (76,99\%, 87/113 -celda A1- versus 23,01\%, 26/113 -celda B1). En el HCBA se encontró que el contexto narrativo favorecía la elección de entonces en un 81,19\%, 82/101 frente a un 18,81\%, 19/101 de elección de así que. En la lectura vertical de la Columna 2 de la misma tabla se ve también que el contexto argumentativo no favorece ninguna de las dos formas en particular ya que estas presentan un 52\%, 52/100 versus un 48\%, 48/100 en las celdas A2 y B2 respectivamente. Algo semejante mostró el cuerpo de datos del HCBA donde se encontró que el contexto argumentativo no favorecía en particular a ninguna de las dos formas: $49,02 \%, 75 / 153$ para entonces versus $50,98 \%, 78 / 153$ para así que.

Resulta también muy interesante la lectura horizontal de la misma Tabla 2 porque da cuenta del contexto que más favorece en particular a cada uno de los conectores: el contexto narrativo es propicio para la elección de entonces (celda A1 67,59\%, 87/139), mientras que el argumentativo lo es para así que (celda B2 64,86\%, 48/74). Es decir que la mayor parte de los usos de entonces se da en contexto narrativo, mientras que la mayor parte de los usos de así que se da en contexto argumentativo ${ }^{19}$.

\footnotetext{
${ }^{19}$ Es interesante el caso siguiente de causa invertida que muestra la adecuación de así que en contexto argumentativo al ser usado como introductor de la apódosis de una oración con cláusula condicional, contexto donde, dada la presencia de una prótasis con $s i$, los gramáticos esperarían entonces:

-¿Vuelven a veces?
} 


\subsection{1. ¿Son los contextos narrativos de entonces iguales que los de así que?}

La observación de la Tabla 2 nos permite ver que si bien en contexto narrativo con conexión consecutiva entre las cláusulas prevalece la elección de entonces, hay un porcentaje no desdeñable de casos de así que que conviven con el relato. Desde el punto de vista metodológico, se espera que se expliquen todos los usos para dar mayor fuerza a las hipótesis defendidas y porque de esa manera se da cuenta de qué fenómeno contextual es el que pesa más en cada caso. Dado esto, el presente apartado bucea dentro de los fragmentos narrativos buscando si los conectores llegan a constituirse en marcas de cesura superestructural. Se siguen los planteos de van Dijk (1978: 143 y 153 a 158) para el análisis de la superestructura de los fragmentos narrativos. Se resumen brevemente sus propuestas, en el marco de los cuales se llevó a cabo el análisis y se explican los ejemplos. Como sabemos, para van Dijk, la coherencia lineal o microestructural y la coherencia global (macro-estructural y superestructural), que reviste características jerárquicas, se asignan al texto.

La coherencia microestructural, que da cuenta de la relación lineal entre proposiciones, presenta tres factores que permiten fundamentar dicha asignación: la coherencia condicional, la coherencia funcional y la distribución de la información. Estos tres factores funcionando simultáneamente permiten asignar coherencia microestructural a dos o más proposiciones.

La coherencia condicional, en términos de van Dijk (1985), se asigna cuando el ordenamiento de proposiciones en secuencias responde a conexiones por relaciones temporales o de causa/resultado, que, en la superficie, queda señalizada por identidad o semejanza léxica, artículos indefinidos/definidos, correlaciones entre los tiempos verbales, el orden de palabras, el orden de las oraciones, el uso de pronombres o adverbios que anafórica o catafóricamente permiten recuperar designados, y el uso de conectores que conectan estados de hechos.

Corresponde ahora atender a la coherencia superestructural de la narración, que es el género textual al que responden los fragmentos bajo análisis en este apartado. Van Dijk reconoce dentro de la superestructura narrativa distintas funciones a asignar a las macro-proposiciones. Las funciones donde prevalecen las acciones de personas constituyen la Historia (que se opone a la Moraleja, entendida como conclusión práctica). La

-Sí, vuelven siempre, no a veces. Si vuelven a Buenos Aires vuelvenn a mi casa, seguro, salvo que vengan en otras condiciones, digamos, vengan con pareja, hijos eh... pero a veces con pareja han vuelto también a casa, SI vuelven ASÍ QUE debe ser bueno el lugar. [PRESEEA-BAIRES J/E3] 
Historia se abre en la Trama y la Evaluación (reacción del narrador frente a la trama). La Trama queda constituida por uno o más Episodios. Cada Episodio está formado por un Marco (donde se presentan el tiempo, el lugar y las circunstancias) y por un Suceso, resultado de la Complicación (como secuencia de acciones), y por la Resolución o Coda (que soluciona el conflicto y cierra la secuencia de acciones de la Complicación).

A partir de estos conceptos, se analizaron los contextos que rodeaban a cada forma bajo estudio dentro del cuerpo de datos y se detectaron dos contextos diferenciados. Uno muestra proposiciones, dentro de la Historia, como parte de la Trama, donde el conector aparece reforzando la secuencia de acciones en la Complicación de un Suceso. A ese contexto se lo llamó "continuativo" o "ilativo" (retomando el término de la NGLE) y se lo mide en la Tabla 3, en la columna 1. El otro contexto detectado donde se daban las formas es la cesura superestructural de la Resolución, donde se inicia la Resolución de un Suceso. Este contexto se mide en la Tabla 3, en la columna 2. Como los fragmentos dentro de los cuales aparecen las dos formas bajo estudio son narrativos, los totales considerados en la Tabla 3, son los correspondientes a la columna 1 de la Tabla 2: 87 casos de entonces y 26 de así que.

El ejemplo (14) muestra la forma entonces funcionando entre proposiciones, dentro de la Historia, como parte de la Trama, conectando la secuencia de acciones en la Complicación de un Suceso. Es importante tener presente que entonces no marca el inicio de la Complicación, es decir que no está funcionando como una marca de cesura superestructural, esto lo diferencia fuertemente del conector con el que se lo está comparando.

En (14) entonces funciona como un conector de estados de hechos, en la Complicación de un Suceso. Complicación en la que marca conexión temporal de anterioridad $>$ posterioridad (se recibió en el Colegio $>$ le quedó una materia sin aprobar $>$ rindió el examen de dicha materia > no pudo empezar los estudios en la Facultad); secuencia reforzada por la correlación de los tiempos verbales (todos los verbos están en pretérito simple), por el orden de las palabras y de las cláusulas que se acumulan ordenadas icónicamente con el orden de los hechos de izquierda a derecha, y además, como decíamos, por la presencia de conectores, dado que encontramos tanto las formas $y$ como entonces, la que no ha perdido totalmente su valor temporal. Se advierte también que hay una relación no solamente temporal entre las proposiciones conectadas por entonces, sino que identificamos claramente una relación de causa/resultado. Sin embargo, lo interesante y lo que en particular nos preocupa destacar aquí por ser la característica que fundamentalmente diferencia entonces de así que, es que entonces no está señalando una parte de la superestructura, sino que, todo lo contrario, está participando de la cadena de acciones. Entre rindió [mal la materia] y no pudo empezar [el Ciclo Básico Común -curso de ingreso a la Universidad de Buenos Aires-] hay solamente relación de 
causa real (anterioridad)/resultado (posterioridad) en los hechos, no estamos ante una cesura superestructural donde se pase de la Complicación a la Resolución. En relación al factor distribución de la información (tercer factor para asignar coherencia condicional), a izquierda y a derecha de entonces se presenta información nueva. Véase (14) como ejemplo de la celda A1 de la Tabla 3.

A diferencia de lo previamente explicado, en (15) se ve claramente que la forma así que señaliza el inicio de la Coda o Resolución, donde hay cambio de tiempo verbal (de pretérito simple - agarró; sacó- a presente - tiene-, forma reforzada por la presencia de un adverbio temporal -ahora-). Es interesante también cómo el hablante vuelve a la secuencia de acciones retomando el pretérito simple: le di mi celular. El caso (15) ejemplifica la celda B2 de la Tabla 3.

(14) [...] se recibió el año pasado y bueno le quedó una materia que la rindió mal, ENTONCES no pudo empezar a hacer el CBC en la primer mitad del año, y se anotó en el UBA XXI, rindió... no sé qué materia rindió un parcial que se sacó un ocho bien, y en el segundo no sé. [PRESEEA_BAIRES SS 1/27]

(15) - Y sí porque es hora de siesta no hay nadie.

-Claro, la agarró un tipo así le dice hacé que me conocés, qué se yo, qué tenés, tenés plata, el celular todo, viste... y le sacó el celular, ASÍ QUE ahora tiene mi celular, le di mi celular que es un celular que no tiene nada. [PRESEEA_BAIRES SS7]

La lectura vertical de la Tabla 3 nos permite decir que en contexto narrativo el hablante prefiere elegir entonces cuando intenta mantener la continuidad en la narración (la celda A1 presenta 93,83\%, 76/81 de los casos de entonces y así que en ese contexto), mientras que prefiere elegir así que en la cesura superestructural para marcar la Coda o Resolución (la celda B2 presenta el 65,62\%, 21/32 de los casos de así que y de entonces en ese contexto $)^{20}$. Domínguez no menciona así que entre los marcadores discursivos de cierre narrativo (2010: 376 y ss.). La lectura horizontal de la misma Tabla 3 corrobora estos resultados, porque el 87,36\%, 76/87 (Celda A1) sobre el total de los

\footnotetext{
${ }^{20}$ En el ejemplo siguiente se ve un interesante contraste entre el uso argumentativo de entonces en un marco de descripción de hechos "siempre que sean hechos excepcionales, festejos familiares entonces se hacen en domingo" versus la conclusión evaluativa "hay muchas tareas, así que el domingo es corto":

- [...] el sábado todo lo que quieran, el domingo salvo algún caso excepcional, algún cumpleaños de familia o algo que nos haya quedado pendiente de no poder hacer el sábado ENTONCES se hace el domingo, pero siempre pensando en volver temprano a casa porque hay que terminar tareas, revisar las mochilas, planchar los guardapolvos, ASÍ QUE el domingo es medio corto... y preparar por supuesto la ropa para toda la semana, ¿no? que no es... [PRESEEA_BAIRES SS36]
} 
casos de entonces en fragmentos con intención narrativa se dan en el desarrollo de los hechos, mientras que así que acumula la mayor parte de sus usos 80,77\%, 21/26 (Celda B2) sobre el total de sus casos en estos fragmentos, en el lugar de la cesura superestructural.

\begin{tabular}{|c|c|c|c|}
\hline & $1+$ Continuidad & $2+$ Coda o resolución & \\
\hline \multirow[t]{3}{*}{ A Entonces } & $76^{21}$ & $11^{22}$ & 87 \\
\hline & $87,36 \%$ & $12,64 \%$ & $100 \%$ \\
\hline & $93,83 \%$ & $34,38 \%$ & \\
\hline \multirow[t]{5}{*}{ B Asíque } & $5^{23}$ & 21 & 26 \\
\hline & $19,23 \%$ & $80,77 \%$ & $100 \%$ \\
\hline & $6,17 \%$ & $65,62 \%$ & \\
\hline & 81 & 32 & 113 \\
\hline & $100 \%$ & $100 \%$ & \\
\hline
\end{tabular}

Odds ratio: $29 \quad X^{2}: 45,84(p>0,05)$

Tabla 3. Los conectores en contexto narrativo

Los resultados descritos para el PRESEEA y para el HCBA nos hacen ubicar la forma entonces no como ordenador discursivo, según analiza Domínguez, sino como un conector continuativo o ilativo entendido en tanto conector de acciones sucesivas. Es decir que, si bien coincidimos con la autora y con otros estudiosos reseñados previamente en cuanto a que entonces interviene en la progresión temporal (y agregamos de causa/resultado) de la narración, disentimos en que en los fragmentos narrativos conversacionales la mayor parte de los usos funcione como ordenador discursivo, dado que

${ }^{21}$ Algunos de estos casos, que por el momento no se tratan por separado porque los totales que se están manejando en este trabajo son reducidos, podrían analizarse como marcas que permiten solamente mantener el canal abierto, partículas fáticas. En Borzi (1997) sobre el HCBA se registró un $12,16 \%, 27 / 222$ del total de casos de entonces que se caracteriza por presentar marcas léxicas y prosódicas de duda en el entorno y cambio de (sub)tópico.

${ }^{22}$ Ejemplo de esta celda:

- [...] porque en ese momento había menos tránsito y la propia avenida Coronel Díaz era un buen, una buena vía de escape, ENTONCES era muy común que se robara allí...

- Mirá vos, me estás dando miedo, ahora que estoy acá... [PRESEEA_BAIRES SS29]

${ }^{23}$ Ejemplo de esta celda: cuando tengo alguna idea todavía la escribo y ayer estaba harta de hacer correcciones a la tesis, ASÍ QUE eh... dejé todo y me puse a escribir un cuento. [PRESEEA_BAIRES J/E1] 
se espera que el ordenador organice superestructuralmente el discurso ${ }^{24}$. La mayor parte de los usos de entonces (que se asemejan además a los ejemplificados por Domínguez) se mantienen a nivel de la coherencia lineal, local o microestructural y esa es su función más típica. Hay solamente un 12,64\%, 11/87 de casos de entonces funcionando en cesura superestructural, no es esta su función más típica en la narración. Lo previamente descrito también sirve para mostrar la ventaja de cuantificar. No basta con partir de casos auténticos, sino que sobre dichos casos corresponde determinar cuál es el caso que prototípicamente representa la función que los hablantes prefieren para esa forma en ese contexto y es para determinar esa preferencia para lo que resulta útil el uso de la cuantificación.

Por el contrario, la forma así que sí se presenta típicamente en cesura superestructural en la narración y sí ordena el discurso.

\subsection{Acumulación con otro conector de la misma orientación semántica y funcional}

Como dijimos al inicio del trabajo (Cf. Apartado 2), el enfoque presupone que el hablante no siempre busca economía en la construcción del discurso, en el sentido de usar una sola marca para cada función. El uso depende (entre otras cosas), por un lado, del interés del hablante por lograr su objetivo comunicativo y, por otro, de la evaluación de la situación que lo llevará a reforzar o no un señalamiento determinado. En consecuencia es lícito preguntarnos ahora si se registra alguna marca en particular que ponga más en evidencia para el interlocutor la intención continuativa que favorece la elección de entonces en contexto narrativo, ya que por el contrario parece que es la búsqueda de señalamiento de un límite lo que guía el uso de así que en esos contextos.

En el presente apartado se estudia si los conectores bajo estudio se acumulan con otros conectores y, si es así, cuál es el aporte que se logra con dicha acumulación. Domínguez (2010: 370) señala que entonces es uno de los marcadores más frecuentes y que se acumula con $y$. Justifica acertadamente esta acumulación citando a Briz (1993: 41) quien afirma que $y$ coadyuva en la función narrativo-progresiva. Domínguez sostiene que desempeñan la misma función y que podría anularse uno de estos, creemos perti-

\footnotetext{
${ }^{24}$ No lo es dada la clasificación de Zorraquino y Portolés, en la que los ordenadores se espera que organicen la información. Tampoco funcionaría entonces en contexto narrativo prototípicamente como un marcador metadiscursivo en la clasificación de Briz (1998) porque no controla ni organiza el mensaje. Es decir que en fragmentos narrativos, aunque haya ciertos casos que sí lo hagan (un 12,64\%, 11/87 -celda A2 de la Tabla 3-) no es su función típica demarcar ni ordenar la conversación, ni señalar las partes del discurso ni su estructura.
} 
nente en este caso señalar el grado de énfasis mayor que favorece la acumulación, y aclarar que los contextos donde entonces se acumula con $y$ no son idénticos a aquellos en los que no se acumulan, aunque solo o con $y$ se trate siempre de fragmentos narrati$\operatorname{vos}^{25}$. Los contextos en los que hay acumulación muestran distinto equilibrio a izquierda y a derecha de los conectores que aquellos en los que no hay acumulación. Un poco más adelante desarrollamos esta concepción de la coordinación como relación discursiva y la importancia que esta conceptualización otorga al equilibrio entre los conectados. Corresponde decir también que Domínguez no explica cómo afectaría esta acumulación al principio de economía que subyace a las teorías y en particular a las teorías gramaticales, dado que se trata aquí de un conector. En el cuerpo de datos bajo análisis, en contexto narrativo, se encontró que había una acumulación recurrente de entonces con $y$, algo que, como muestra la Tabla 4, no se da en el mismo contexto con así que, el que no se acumula con ninguna otra forma ni cuando coincide en contexto continuativo ni cuando marca coda narrativa. La conjunción copulativa, prototípica de la relación de coordinación, une constituyentes que presentan un alto equilibrio semántico, pragmático, sintáctico y prosódico a izquierda y a derecha del conector, con la única ruptura que da el hecho de que, en la linealidad, un constituyente (una cláusula en nuestro caso) está ubicado posicionalmente antes que el otro $^{26}$. En lo referente al equilibrio semántico entre los conectados, remitimos al trabajo pionero de Lang (1977), quien postula que el interlocutor construye un Integrador Común o Marco de Integración Global para unir en sus semejanzas los conectados de una coordinación ((compró) un auto y una moto > (compró) vehículos). En lo referente a la calidad de la información de los conectados y a la localización de los respectivos acentos principales, ambos conectados coordinados tienden a presentar información nueva señalada con el acento principal del constituyente ${ }^{27}$. Los constituyentes clausales coordinados presentan dos acciones encadenadas o sucesos de un mismo episodio con igual importancia en el discurso que cumplen a su vez igual función retórica, lo que redunda en equilibrio semántico-pragmático. Los tiempos y el modo verbal de ambos constituyentes tienden a ser idénticos y las cláusulas están ordenadas icónicamente según el orden de los hechos favoreciéndose una interpretación de anterioridad / posterioridad en los casos no marcados. El caso (16) presen-

\footnotetext{
${ }^{25}$ No se desarrolla este análisis en el presente trabajo, porque compete a la descripción de la forma entonces comparada consigo misma, es decir, la descripción de todos los contextos de uso de esa forma y no aquellos en los que varía con así que o con otro marcador o conector.

${ }^{26}$ Véase al respecto la propuesta de Haiman (1985) y una aplicación a casos con entonces del español de la Argentina en Borzi (1997: 149-153).

${ }^{27}$ Esta característica fue enunciada para el español por Contreras (1978).
} 
ta un contexto semejante al descripto; ejemplifica la celda A1, que en la lectura vertical de la Tabla 4 muestra en comparación con así que un 100\% de las ocurrencias en dicha celda.

(16) -[...] No, qué primera, ya era deep blue, se llamaba "deep blue", era una computadora muy moderna y jugó una partida con Kasparov, que era el campeón mundial de ajedrez, y le ganó, la computadora. Y ENTONCES yo escribí un editorial para el diario en ese momento que se titulaba: “¿Estás contenta, Deep Blue?" y el editorial trataba. [...] [PRESEEA_BAIRES JM12]

\begin{tabular}{|c|c|c|c|}
\hline & $1+y$ & $2-y$ & \\
\hline \multirow[t]{3}{*}{ A Entonces } & 24 & 63 & 87 \\
\hline & $27,59 \%$ & $72,41 \%$ & $100 \%$ \\
\hline & $100 \%$ & $70,79 \%$ & \\
\hline \multirow[t]{5}{*}{ B Asíque } & 0 & 26 & 26 \\
\hline & $0 \%$ & $100 \%$ & $100 \%$ \\
\hline & $0 \%$ & $29,21 \%$ & \\
\hline & 24 & 89 & 113 \\
\hline & $100 \%$ & $100 \%$ & \\
\hline
\end{tabular}

Tabla 4. Acumulación de las formas bajo estudio con ' $y$ '

La lectura vertical de la columna 1 de la Tabla 4 es contundente, entonces se acumula con $y$, un conector de su misma orientación semántica, cumpliendo una función ilativa o continuativa conectando una secuencia de acciones o sucesos de un mismo episodio. En el HCBA también se dio una celda cero en relación con así que. En el HCBA hay un porcentaje también importante sobre todos los casos de entonces que lo muestran combinado con $y, 17,12 \%, 38 / 222$, aunque este es menor que el que se presenta en el cuerpo de datos bajo análisis aquí.

\section{Conclusiones}

Los resultados obtenidos sobre parte del PRESEEA_BAIRES y los obtenidos sobre el HCBA nos permiten afirmar que tanto entonces como así que varían en contexto consecutivo en el español hablado de Buenos Aires. Como se dijo, un estudio contrastivo empírico cuali-cuantitativo, que diferencia los contextos, nos orienta hacia una descripción sistemática de dichas formas en tanto permite identificar con qué significados y en qué funciones prefiere usar el hablante más frecuentemente cada una de estas. 
En primer lugar, como se concluyó en Borzi (1997) sobre datos del HCBA, encontramos que en el PRESEEA_BAIRES, a la manera de los marcadores discursivos, entonces funciona como tematizador de información y así que como marca de abandono de turno. En esos contextos, entonces activa su significado mostrativo temporal anafórico (significado registrado por la NGLE, 2009: \$17.9g-i), mientras que así que "constituye un límite hacia adelante" y expresa posterioridad (NGLE, 2009: \$23.16k) o catáfora (NGLE, 2009: \$17.9k).

Se puede afirmar también que en contexto de intención narrativa, entonces es un conector que preferentemente conecta secuencias de acciones o estados de hechos, dentro de la Complicación. Por su parte, así que, también en contexto narrativo, focaliza la Coda o Resolución. Se sostiene, de esta manera, que preferentemente entonces funciona como conector continuativo o ilativo, no como ordenador del discurso, mientras que sí funciona como ordenador discursivo así que, porque interviene señalizando la superestructura narrativa. En estos contextos entonces no solamente activa su significado mostrativo temporal de anterioridad, simultaneidad y posterioridad para intervenir en contextos de sucesión temporal, sino también para intervenir en contextos de causa real/resultado.

Si bien no se tratan en este trabajo los contextos argumentativos sobre el PRESEEA, sí podemos decir que según resultados de Borzi (1997) (sobre discurso oral) y de Borzi (2004) (sobre discurso escrito) es en fragmentos argumentativos donde más prototípicamente entonces orienta la información de izquierda a derecha y ordena el discurso, interviniendo en la señalización de la superestructura argumentativa.

Digamos por otro lado que sobre el total de casos de entonces analizados (100\%/157) se registró 1 caso (un 0,64\%, 1/157) en abandono de turno; 17 casos en contexto de tematización $(10,82 \%, 17 / 157), 87$ casos en contexto narrativo $(55,42 \%, 87 / 157)$ y 52 en argumentativo $(33,12 \%, 52 / 157)$, estos últimos no sub-analizados en el presente trabajo.

Sobre el total de casos de así que (100\%/100) se registraron 20 casos en contexto de abandono de turno, 6 casos ecoicos, 26 en contexto de intención narrativa y 48 en contexto de intención argumentativa, estos últimos tampoco fueron sub-analizados en el presente trabajo.

El análisis contrastivo cualitativo y cuantitativo nos permitió aportar evidencia respecto de los contextos de uso más típicos de cada uno de los dos conectores y mostrar que no son intercambiables entre sí y que siempre aportan significado. 


\section{Referencias bibliográficas}

Alcina Franch, Juan y José Manuel Blecua (1975): Gramática Española. Barcelona, Ariel.

Álvarez Menéndez, Alfredo (1991): "Conectores y grupos oracionales consecutivos", Lingüística Española Actual, 13/1, págs. 117-132.

Álvarez, Alfredo. (1995): Las construcciones consecutivas. Madrid, Arco/Libros.

Álvarez, Alfredo (1999): "Las construcciones consecutivas". En Bosque, Ignacio y Violeta Demonte, (dirs.), págs. 3739-3804.

Beinhauer, Werner (1978): El español coloquial. Madrid, Gredos.

Bello, Andrés (1980) [1847]: Gramática de la lengua castellana. Madrid, EDAF.

Borzi, Claudia (1997): Syntax, Semantik und Pragmatik der Konnektoren entonces, así que und (tal/..) que (Diss.). Ludwig-Maximilians-Universität, Munich, Profidruck.

Borzi, Claudia (2004): "Sintaxis de entonces en corpus escrito". En Arnoux, Elvira y María Marta García Negroni (eds.), Homenaje a Oswald Ducrot. Buenos Aires, EUDEBA, págs. 47-76.

Bosque Ignacio y Violeta Demonte, (dirs.) (1999): Gramática Descriptiva de la Lengua Española, Madrid, Espasa.

Briz Gómez, Antonio (1993). Los conectores pragmáticos en la conversación coloquial (II): su papel metadiscursivo. Español Actual 59, 39-56.

Briz Gómez, Antonio (1998): El español coloquial en la conversación. Esbozo de pragmalingüística. Barcelona, Ariel.

Briz, Antonio, Salvador Pons Bordería y José Portolés (coords.): Diccionario de partículas discursivas del español. http://www.dpde.es/

Casado Velarde, Manuel (1998): "Lingüística del texto y marcadores del discurso". En Martín Zorraquino, María Antonia y Estrella Montolío Durán (eds.), Marcadores del discurso. Madrid, Arco/Libros, págs. 55-70.

Casares, Julio (1979): Diccionario ideológico de la lengua española. Barcelona, G. Gili.

Contreras, Heles (1978): El orden de palabras en español. Madrid, Cátedra.

Cortés Rodríguez, Luis (1991): Sobre conectores expletivos y muletillas en el español hablado. Málaga, Editorial Librería Ágora.

Domínguez García, María Noemí (2010): "Los marcadores del discurso y los tipos textuales”. En Loureda Lamas, Óscar y Esperanza Acín Villa (coords.). Los estudios sobre marcadores del discurso en español, hoy. Madrid, Arco/Libros, págs. 359-413.

Fernández Ramírez, Salvador (1986): Gramática Española. 4. El verbo y la oración. Madrid, Arco/Libros.

Firbas, Jan (1992): Functional Sentence Perspective in Written and Spoken Comunica- 
tion. Cambridge, Cambridge University Press.

Fuentes Rodríguez, Catalina (1987): Enlaces Extraoracionales. Sevilla, Alfar.

García, Erica (1985): “Shifting variation”, Lingua, 67, págs. 189-224.

García Fernández, Luis (1999): "Los complementos adverbiales temporales. La subordinación temporal”. En Bosque, Ignacio y Violeta Demonte (dirs.), págs. 3129-3208.

Gili Gaya, Samuel (1961): Curso Superior de Sintaxis Española. Barcelona, Biblograf.

Haiman, John (1985): “Symetry”. En Haiman, John (ed.), Iconicity in Syntax. Amsterdam, John Benjamins, págs. 73-75

Kany, Charles (1969): Sintaxis hispanoamericana. Madrid, Gredos.

Koch Peter \& Wulf Oesterreicher (1990): Gesprochene Sprache in der Romania: Französisch, Italienisch, Spanisch. Tübingen, Niemeyer.

Kovacci, Ofelia (1990/92): El comentario gramatical, vols. 1 y 2. Madrid, Arco/Libros.

Lakoff, George (1987): Women, fire and dangerous things. Chicago, University Press.

Lang, Ewald (1977): Semantik der koordinativen Verknüpfung. Berlin, Akademie Verlag

Langacker, Ronald (1987): Foundations of Cognitive Grammar. Theoretical Prerequisites, vol. 1. Stanford, Stanford University Press.

Langacker, Ronald (1991): Foundations of Cognitive Grammar. Descriptive Application, vol. 2. Stanford, Stanford University Press.

Lenz, Rodolfo (1935): La oración y sus partes. Madrid, Nueva Revista de Filología Española.

Lope Blanch, Juan M. (1984): “Anomalías en el habla popular de México”. En Schwartz de Lerner, Lía e Isaías Lerner (eds.), Homenaje a Ana María Barrenechea. Madrid, Castalia, págs. 99-104.

Martín Zorraquino, María Antonia y José Portolés Lázaro (1999): "Los marcadores del discurso". En Bosque, Ignacio y Violeta Demonte (dirs.), págs. 4051-4213.

Martínez, Angelita (2009): "Metodología de la investigación lingüística: el enfoque etnopragmático". En Narvaja de Arnoux, Elvira (dir.), Escritura y producción de conocimiento en las carreras de posgrado. Buenos. Aires, Santiago Arcos editor, págs. 259-286.

Matte Bon, Francisco (1992): Gramática comunicativa del español, vol. 2. Madrid, Difusión.

Moliner, María (1981): Diccionario de uso del español. Madrid, Gredos.

Pereira Scherre, María Marta y Anthony Julius Naro (1991): "Marking in discourse: 'Birds of a feather", Language Variation and Change, 3, págs. 23-32.

Pottier, Bernard (1962): Systématique des éléments de relation. Étude de morphosyntaxe structurale romane. Paris, Klincksieck.

Real Academia Española (1931): Gramática. Madrid, Espasa Calpe. 
Real Academia Española (1973): Esbozo de una Nueva Gramática de la Lengua Española. Madrid, Espasa Calpe.

Real Academia Española (2009): Nueva gramática de la lengua española. Madrid, Espasa.

Real Academia Española (1984): Diccionario de la lengua española. Madrid, Espasa Calpe.

Roca Pons, José (1960): Introducción a la gramática. Barcelona, Vergara.

Seco, Rafael (1978): Manual de gramática española. Madrid, Aguilar.

Seco, Manuel (1989): Gramática esencial del español. Madrid, Aguilar.

Van Dijk, Teun A. (1980) [1978]: La ciencia del texto. Buenos Aires, Paidós,

Van Dijk, Teun A. (1985): “Semantic Discourse Analysis”. En Van Dijk, Teun A. (ed.),

Handbook of discourse analysis - Dimensions of Discourse. Orlando, Florida, págs. 103-136.

Veciana, Roberto (1984): “'Entonces’, sus usos”, Yelmo, 60/61, págs. 12-23.

Vigara Tauste, Ana María (1992): Morfosintaxis del español coloquial. Madrid, Gredos. 